\title{
Estudo comparativo do óleo essencial de Eugenia punicifolia (HBK) DC. de diferentes localidades de Pernambuco
}

\author{
R.N. de Oliveira', I.J.M. Dias ${ }^{2}$, C.A.G. Câmara ${ }^{2 *}$ \\ ${ }^{1}$ Departamento de Química Fundamental, Universidade Federal de Pernambuco, Av. Morais Rego s/n, Cidade \\ Universitária, 50670-901, Recife, PE, Brasil, \\ ${ }^{2}$ Departamento de Química, Universidade Federal Rural de Pernambuco, Rua Dom Manoel de Medeiros s/n, 52171 - \\ 900, Recife, PE, Brasil
}

\begin{abstract}
RESUMO: O óleo essencial obtido por hidrodestilação das folhas de Eugenia punicifolia, coletados a partir de duas regiões de Matas Serranas do Estado de Pernambuco (Serra Negra e Brejo da Madre de Deus), foi analisado por CG/EM. O monoterpeno oxigenado, linalol (SN: 44,0\%, BMD: 61,2\%), foi o componente principal do óleo de ambas amostras. Cerca de $66,2 \%$ da constituição dos voláteis da amostra proveniente de $\mathrm{SN}$ e 74,2\% de BMD referem-se aos monoterpenos oxigenados, enquanto $33,6 \%$ da amostra de $\mathrm{SN}$ e $20,5 \%$ de BMD são sesquiterpenos oxigenados, sendo o $\beta$-cariofileno o mais abundante (SN: 22,7\%, BMD: 16,2\%). Com exceção do $\alpha$-cadinol, presente apenas no óleo proveniente do Brejo da Madre de Deus, a constituição química de ambas as amostras foi à mesma, variando apenas o percentual relativo de seus constituintes.
\end{abstract}

Unitermos: Eugenia punicifolia, Myrtaceae, óleo essencial, linalol, $\beta$-cariofileno, Matas Serranas.

\begin{abstract}
Comparative study of the essential oil of Eugenia punicifolia (HBK) DC. from diferent places of Pernambuco". The essential oils obtained from the leaves of Eugenia punicifolia by hydrodistillation, which were collected from two different locations of Pernambuco (Serra Negra and Brejo da Madre de Deus), have been investigated by GC/MS. Linalol was found to be the main component of this oil from SN $(44,0 \%)$ and BMD $(61,2 \%)$. About $66,2 \%$ of the oil constituents from SN and 74,2\% from BMD are oxygenated monoterpenes, with predominance of $\beta$-cariofileno (SN: 22,7 and BMD: 16,2\%). With exception of $\alpha$-cadinol, present only in the oils from plants colleted in Brejo da Madre de Deus, the chemical composition of both samples was quite similar, the only difference was the relative content among their components.
\end{abstract}

Keywords: Eugenia punicifolia, Myrtaceae, essential oil, linalol, $\beta$-caryophyllene, highland bushes.

\section{INTRODUÇÃO}

Apesar de topograficamente se encontrarem dentro da Zona da Caatinga, as formações florestais do Nordeste brasileiro, que ocorrem em encostas e topos de serras com altitudes superiores a $500 \mathrm{~m}$ são denominadas de Matas Serranas ou Brejos de Altitudes. Em Pernambuco, há registro de pelo menos 11 destas formações florestais (Sobrinho, 1970). Os gêneros e espécies que habitam estas formações florestais, localizadas em Pernambuco na parte oriental do planalto da Borborema, estão relacionados diretamente com táxons que ocorrem na Amazônia. É o caso das espécies do gênero Eugenia, que na região Norte são conhecidas popularmente como murta, pedra-ume e pedra-ume-caá (Maia et al., 1997).

Com 3000 espécies e cerca de 80 gêneros, a Myrtaceae se destaca como uma das famílias mais importantes devido sua ampla distribuição em todos os ecossistemas brasileiros (Fabris; Cesar, 1996), sendo o gênero Eugenia L. um dos maiores, com mais de 500 espécies, das quais cerca de 400 encontram-se no Brasil e assumem destaque especial por serem utilizadas como plantas medicinais. A pesar de cientificamente não ter sido verificada tal propriedade, algumas espécies são utilizadas na medicina popular, principalmente no tratamento de ferimentos e infecções intestinais (Adebajo et al., 1989). Por outro lado, há pesquisas que confirmam a presença de substâncias com potencial para uso medicinal. É o caso da decocção de folhas de $E$. uniflora, ricas em flavanóides, utilizada no controle da hipertensão e da gota (Schmeda-Hirschmann et al., 1987; Theoduloz et al., 1988); e a atividade anticonvulsante do óleo essencial de E. caryophyllata (Pourgholami et al., 1999) a qual está relacionada aos constituintes eugenol e carvacrol (Dallmeier; Carlini, 1981; XI et al., 1994). Trabalhos mais recentes são encontrados na literatura reportando o potencial antimicrobiano do óleo essencial das folhas de E. stipitata McVaugh ssp. sororia (Medeiros et al., 2003) e E. dysenterica (Costa et al., 2000), bem como de outras partes da planta (Larhsini et al., 2001; 

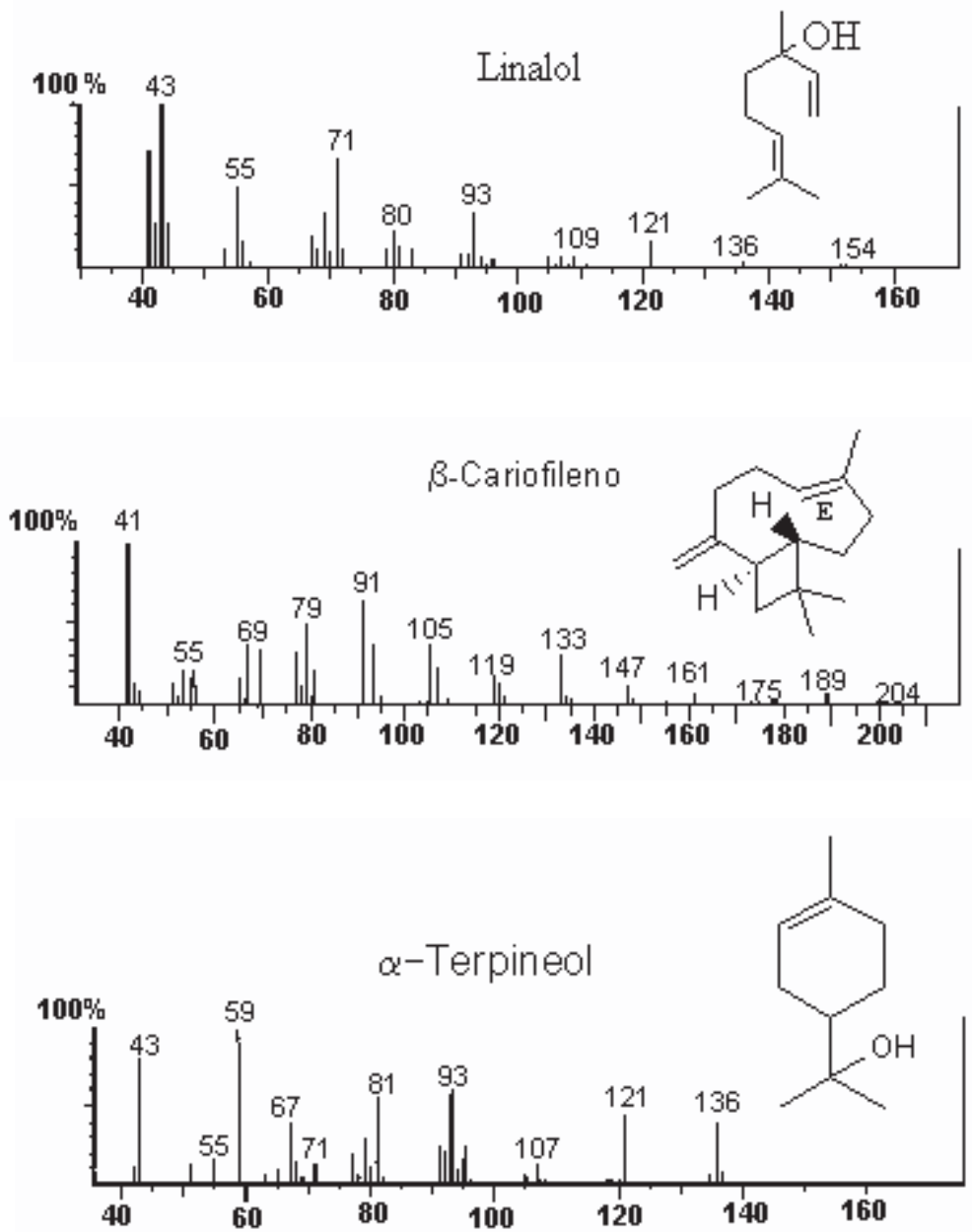

Figura 1. Espectro de massas dos componentes majoritários identificados no óleo essencial das folhas de E. punicifolia das regiões de Matas Serranas de Pernambuco

Holetz et al., 2002; Rajkumar; Berwal, 2003; De Souza et al., 2004). Há outros trabalhos relacionados com as atividades: inseticida (Kokate; Chintalwar, 2003; Yang et al., 2003) e acaricida (Kim et al., 2003) do óleo essencial de E. caryophyllata.

Em visita às comunidades de Serra Negra (SN) e Brejo da Madre de Deus (BMD), alguns moradores relataram a utilização de infusões alcoólica de folhas de E. punicifolia para o tratamento de ferimentos e doenças infecciosas.

Levantamento bibliográfico sobre o estudo fitoquímico da espécie E. punicifolia revelou apenas uma citação referente à constituição química do óleo essencial das folhas provenientes de duas regiões próximas à Manaus. Em ambas as amostras, o $\beta$-cariofileno (amostra "A": $32,9 \%$ e B: $23,6 \%$ ) foi o constituinte majoritário (Maia et al., 1997).

Neste trabalho reportamos a composição química do óleo essencial das folhas de E. punicifolia nativas de duas localidades de Matas Serranas de Pernambuco e comparamos com as de outras regiões do Brasil.

\section{MATERIAL E MÉTODOS}

Folhas frescas de E. punicifolia (ABK) foram coletadas em setembro de 2001 nas Matas Serranas do estado de Pernambuco conhecidas por Serra Negra (SN) e Brejo da Madre de Deus (BMD). A exsicata do material botânico coletado foi depositada sob o registro LSS $n^{\circ}$ 2633 no Herbário Vasconcelos Sobrinho da Universidade Federal Rural de Pernambuco.

As folhas frescas foram submetidas a hidrodestilação em um aparelho doseador do tipo Cleavenger por duas horas. O óleo essencial obtido foi separado e seco com sulfato de sódio anidro. O rendimento foi calculado com base no peso fresco do material vegetal com experimentos em triplicata.

Os óleos obtidos foram analisados por cromatografia gasosa acoplada à espectroscopia de massas utilizando um cromatógrafo HP 5890B SERIES II, acoplado a um espectrômetro de massas HP-5971 equipado com uma coluna capilar de sílica fundida $\mathrm{J} \&$ W Scientific DB5 (30 m x 0,25 $\mu \mathrm{m})$. As temperaturas do 
Tabela 1. Percentual dos constituintes químicos do óleo essencial das folhas de Eugenia punicifolia coletadas em Serra Negra e Brejo da Madre de Deus.

\begin{tabular}{|c|c|c|c|c|}
\hline Compostos & $\begin{array}{c}\text { Índice Kovat } \\
\text { obtido }^{\mathrm{a}}\end{array}$ & $\begin{array}{c}\text { Índice Kovat } \\
\text { literatura }\end{array}$ & Serra Negra & Brejo da Madre de Deus \\
\hline$\alpha$-Pineno & 935 & 927 & $\mathrm{t}^{\mathrm{b}}$ & 1,1 \\
\hline$\beta$-Pineno & 964 & 967 & $\mathrm{t}$ & 1,3 \\
\hline 1,8-Cineol & 1029 & 1033 & 3,6 & 2,9 \\
\hline cis-Oxido de linalol & 1070 & 1074 & 1,4 & 0,6 \\
\hline trans-Oxido de linalol & 1079 & 1088 & 1,7 & 0,5 \\
\hline Linalol & 1082 & 1098 & 44,0 & 61,2 \\
\hline Isoborneol & 1151 & 1156 & 1,0 & $\mathrm{t}$ \\
\hline Terpinen-4-ol & 1172 & 1177 & 3,4 & 2,3 \\
\hline$\alpha$-Terpineol & 1183 & 1189 & 8,8 & 6,7 \\
\hline Geraneato de metila & 1319 & 1323 & 2,3 & $\mathrm{t}$ \\
\hline$\delta$-Cubebeno & 1348 & 1351 & 4,7 & 1,2 \\
\hline$\beta$-Elemeno & 1388 & 1391 & 3,7 & 1,8 \\
\hline$\beta$-Cariofileno & 1415 & 1418 & 22,7 & 16,2 \\
\hline Aromadendreno & 1435 & 1439 & 2,5 & $\mathrm{t}$ \\
\hline Germancreno B & 1475 & 1480 & $\mathrm{t}$ & 1,3 \\
\hline Elemol & 1533 & 1538 & $\mathrm{t}$ & 0,9 \\
\hline$\alpha$-Cadinol & 1574 & 1578 & - & 1,5 \\
\hline Total & & & 99,8 & 99,5 \\
\hline
\end{tabular}

injetor e detector foram respectivamente $290^{\circ} \mathrm{C}$ e $285^{\circ} \mathrm{C}$. $\mathrm{O}$ gás de arraste usado foi hélio com vazão de $1 \mathrm{~mL} /$ min e o programa de temperatura da coluna foi $40^{\circ} \mathrm{C}(1$ minuto) até $220^{\circ} \mathrm{C} \mathrm{a} 4^{\circ} \mathrm{C} / \mathrm{min} ; 220^{\circ} \mathrm{C}\left(0\right.$ minuto) até $280^{\circ} \mathrm{C}$ a $20^{\circ} \mathrm{C} / \mathrm{min}$. Os espectros de massas foram obtidos com um impacto eletrônico de $70 \mathrm{eV}, 0,84 \mathrm{scan} / \mathrm{sec}$ de $\mathrm{m} / \mathrm{z} 40$ a 550. Os compostos foram identificados inicialmente usando a biblioteca dos massas em computador com índices Kovats como pré-seleção de rotina (Alencar et al., 1984), seguida pela confirmação visual de padrões dos massas reportados na literatura (Adams, 1995).

\section{RESULTADOS E DISCUSSÃO}

Os rendimentos dos óleos para as amostras de $\mathrm{SN}$ e BMD foram $0,79 \%$ e $0,82 \%$, respectivamente. Ao todo, 17 compostos foram identificados correspondendo cerca de $99 \%$ do óleo. Em ambas as amostras o linalol (SN: 44,0\% e BMD: 61,2\%) foi o constituinte majoritário, seguido pelo $\beta$-cariofileno (SN: $22,7 \%$ e BMD: $16,2 \%$ ) e á-terpineol (SN: 8,8\% e BMD: 6,7\%) (Figura 1).

A composição química dos óleos das folhas obtidas de diferentes amostras de E. punicifolia (SN e BMD) é apresentada na Tabela 1.

Basicamente, a composição química dos óleos analisados foi semelhante, os quais são constituídos principalmente de monoterpenos oxigenados (SN: 66,2\% e BMD: $74,2 \%$ ) e sesquiterpenos oxigenados (SN: 33,6\% e BMD: 20,5\%). O perfil químico do óleo essencial obtido nas amostras (SN e BMD) foi o mesmo. Ambas as amostras revelaram os mesmos constituintes, com exceção do $\alpha$-cadinol, presente apenas na amostra coletada em BMD. Existe alguns outros componentes no óleo proveniente de BMD, que foram identificados na amostra coletada em SN em uma concentração inferior a $1 \%$, é o caso dos monoterpenos: $\alpha$-pineno, $\beta$-pineno, e dos sesquiterpenos: germancreno $\mathrm{B}$ e elemol. Por outro lado, existem alguns componentes identificados no óleo procedente de $\mathrm{SN}$, que foram encontrados no óleo proveniente de BMD em uma concentração inferior a 1\% ( isoborneol, geraneato de metila e aromadendreno).

Levando em consideração o critério estabelecido por Knudsen et al. (1993) para agrupar constituintes voláteis em diferentes classes químicas, oóleo essencial das amostras de E. punicifolia das regiões de Matas Serranas de Pernamabuco (SN e BMD) revelaram as classes dos monoterpenos e sesquiterpenos como componentes principais. Ou seja, o mesmo perfil observado para a maioria de suas congêneres investigadas (Apel et al., 2004a-d; Vila et al., 2004), só para citar os resultados mais recentes. Algumas exceções são reportadas na literatura para as espécies E. stigmatosa, cujo óleo é constituído basicamente pelo derivado de ácido carboxílico, o ácido (Z)-tetradec-5-enoico (Apel et al., 2004a) e o benzenóide, eugenol que é o componente majoritário do óleo de $E$. caryophyllata (Deyama; Horiguch, 1971).

Por outro lado, comparando os resultados obtidos para o óleo essencial de E. punicifolia das regiões de Matas Serranas com aqueles obtidos a partir de duas amostras foliares de E. punicifolia, coletadas em regiões próximas à Manaus foram constatadas diferenças significativas, principalmente no componente principal. Dos 17 componentes identificados nas amostras provenientes das regiões de Matas Serranas, apenas 7 foram identificados 
nas amostras proveniente da Amazônia.

Utilizando a mesma parte da planta e a mesma metodologia para obtenção do óleo essencial, Maia et al. (1997) encontraram, além de compostos bezenóides, o $\beta$-cariofileno como o constituinte majoritário em ambas às amostras. $O$ percentual de $\beta$-cariofileno $(23,6 \%)$ encontrado na amostra $\mathrm{B}$ foi compatível com o percentual obtido na amostra coletada em SN (22,7\%). Mas, o constituinte majoritário encontrado em SN e BMD foi o linalol com $44 \%$ e $61,2 \%$, respectivamente. O linalol está presente nas amostras A e B em um percentual abaixo de $1 \%$ e nenhuma das congêneres estudadas até o momento tem apresentado o linalol como componente principal, apenas uma espécie nativa de Cuba (E. cristata) revelou a presença de linalol, com percentual, um pouco acima de 6\% (Pino et al., 2001). Por outro lado, o fenilpropanóide, dilapiol, presente na composição do óleo estudado por Maia et al. (1997) não foi observado nas amostras coletadas em Pernambuco (S N e BMD).

Variações na composição química do óleo essencial de uma mesma espécie de regiões distintas, como Amazônia e Matas Serranas de Pernambuco podem ser atribuídas às diferenças dos parâmetros climáticos e geográficos, como temperatura, altitude, direção do vento, pluviosidade, tipo de solo, etc. (Douglas et al., 2004).

\section{CONCLUSÃO}

Os monoterpenos: 1,8-cineol; cis e trans-oxido de linalol; isoborneol; terpinen-4-ol; á-terpineol e os sesquiterpenos: elemol e $\alpha$-cadinol são relatados pela primeira vez na espécie E. punicifolia.

A composição química do óleo essencial entre as amostras de SN e BMD apresentou uma distribuição qualitativa e quantitativa dos constituintes químicos bastante similares. Porém, quando comparado com o óleo essencial de outras regiões do Brasil, revelou diferenças significativas, principalmente no constituinte majoritário e na ausência de componentes benzenóides. As diferenças na composição química do óleo essencial das amostras de E. punicifolia coletadas em diferentes regiões do Brasil sugerem a existência de um novo quimiotipo para a espécie E. punicifolia ocorrendo nas Matas Serranas de Pernambuco. Ou seja, além do quimiotipo â-cariofileno, reportado por Maia et al. (1997) para E. punicifolia que ocorre na Amazônia, foi identificado o quimiotipo linalol (SN: 44,0\% e BMD: 61,2\%), percentual inusitado para a espécie E. punicifolia e suas congêneres.

\section{AGRADECIMENTOS}

Os autores agradecem aos Professores Edilberto R. Silveira e Afrânio Craveiro da Universidade Federal do Ceará pelas análises dos óleos e a FACEPE pelo suporte financeiro.

\section{REFERÊNCIAS}

Adams RP 1995. Identification of essential oil components by gas chromatography/mass spectroscopy. Illinois: USA, Allured Publ. Corp., Carol Stream.

Adebajo AC, Oloke KJ, Aladesanmi AJ 1989. Antimicrobial activity of the leaf and extract of Eugenia uniflora. Phytother Res 3: 258-259.

Alencar JW, Craveiro AA, Matos FJA 1984. Kovats indices as a preselection routine in mass spectra library search of volatiles. J Nat Prod 47: 890-892.

Apel MA, Sobral M, Schapoval EES, Henriques AT, Menut C, Bessiere JM 2004a. Chemical composition of the essential oils of Eugenia hymalis and Eugenia stigmatosa. Part VI: section Biflorae. J Essent Oil Res 16: 437-439.

Apel MA, Sobral M, Schapoval EES, Henriques AT, Menut C, Bessiere JM 2004b. Essential oils composition of Eugenia florida and Eugenia mansoi.J Essent Oil Res 16: 321-322.

Apel MA, Sobral M, Schapoval EES, Henriques AT, Menut C, Bessiere JM 2004c. Chemical composition of the essential oils of Eugenia beaurepaireana and Eugenia pyriformis: Section Dichomatae. J Essent Oil Res 16: 191-192.

Apel MA, Sobral M, Schapoval EES, Henriques AT, Menut C, Bessiere JM 2004d. Essential oils from Eugenia species - Part VII: Sections Phyllocalyx and Stenocalyx. J Essent Oil Res 16: 135-138.

Costa TR, Fernandes OFL, Santos SC, Oliveira CMA, Lião LM, Ferri PH, Paula RJ, Ferreira HD, Sales BHN, Silva MRR 2000. Antifungal activity of volatile constituents of Eugenia dysenterica leaf oil. J Ethonopharmacol 72: 111-117.

Dallmeier K, Carlini EA 1981. Anesthetic, hypothermic, myorelazant and anticonvulsant effects of synthetic eugenol derivatives and natural analogues. Pharmacology 22: 113-127.

De Souza GC, Haas APS, Von Poser GL, Schapoval EES, Elisabesky E 2004. Ethnopharmocolgical studies of antimicrobial remedies in the south of Brasil. $J$ Ethnopharmacol 90:135-143.

Deyama T, Horiguch T 1971. Studies on components of essential oil of clove (Eugenia caryophyllata Thumberg). Yakugaku Zasshi 91: 13831385.

Douglas MH, Van Klink JW, Smallfield BM, Perry NB, Anderson RE, Johstone P, Weavers RT 2004. Essential oils from New Zeland manuka: triketone and other chemotypes of Leptospermum scoparium. Phytochemistry 65: 1255-1264.

Fabris LC, Cesar O 1996. Estudos florísticos em uma mata litorânea no sul do estado do Espírito Santo. Boletim do Museu de Biologia Mello Leitão 5: 15-46.

Holetz FB, Pessini GL, Sanches NR, Cortez DAG, Nakamura CV, Filho BPD 2002. Screening of some plants used in the Brazilian folk medicines for the treatment of infectious diseases. Mem Inst Oswaldo Cruz 97: 1027-1031.

Kim EH, Kim HK, Choi DH, Ahn YJ 2003. Acaricidal activity of clove bud oil compounds against Tyrophagus putrescentiae (acari: Acaridae). Appl Entomol Zool 38: 261-266.

Knudsen JT, Tollsten L, Bergström LG 1993. Floral scents 
- a checklist of volatile compounds isolated by headspace techniques. Phytochemistry 33: 253-280.

Kokate SD, Chintalwar GJ 2003. Insect repellent activity of certain plant extracts against pulse beetle, Callosobruchus chinensis L. Natl Acad Sci Lett 26: 44-46.

Larhsini M, Oumoulid L, Lazrek HB, Wataleb S, Bousaid M, Bekkouche K, Jana M 2001. Antibacterial activity of some Moroccan medicinal plants. Phytother Res 15: 250-252.

Maia JGS, Zoghbi MGB, Luz AIR 1997. Essential oil of Eugenia punicifolia (HBK) DC. J Essent Oil Res 9: 337-338.

Medeiros JR, Medeiros N, Medeiros H, Davin LB, Lewis NG 2003. Composition of the bioactive oils from the leaves of Eugenia stipitata McVaugh ssp. sororia from the Azores. J Essent Oil Res 15: 293-295.

Pino J, Bello A, Urquiola A, Garcia S, Rosado A 2001. Leaf oil of Eugenia cristata Wr. from Cuba. J Essent Oil Res 13: 407-408.

Pourgholami MH, Kamalinejad M, Javadi M, Majzoob S, Sayyah M 1999. Evaluation of the anticonvulsant activity of the essential oil of Eugenia caryophyllata in male mice. J Ethnopharmacol 64: 167-171.

Rajkumar V, Berwal JS 2003. Inhibitory effect of clove (Eugenia caryophyllus) on toxigenic molds. J Food Sci Tech Mys 40: 416-418.

Schmeda-Hirschmann G, Theoduloz C, Franco L, Ferro EB, Arias AR 1987. Preliminary pharmacological studies on Eugenia uniflora leaves: xanthine oxidase inhibitory activity. J Ethnopharmacol 21: 183-186.

Sobrinho JV 1970. As regiões naturais do Nordeste o meio ambiente e a civilização. Recife: Conselho de Desenvolvimento de Pernambuco, p. 441.

Theoduloz C, Franco L, Ferro EB, Schmeda-Hirschmann G 1988. Xanthine oxidase inhibitory activity of Paraguayan Myrtaceae. J Ethnopharmacol 24: 179183.

Vila R, Iglesias J, Canigueral S, Santana AI, Solis PN, Gupta MP 2004. Constituents and biological activity of the essential oil of Eugenia acapulcensis Steud. J Essent Oil Res 16: 384-386.

Xi LY, Zheng WM, Zhen SM, Xian NS 1994. Rapid arrest of seizure with an inhalation aerosol containing diazepam. Epilepsia 35: 356-358.

Yang YC, Lee SH, Lee WJ, Choi DH, Ahn YJ 2003. Ovicidal and adulticidal effects of Eugenia caryophyllata bud and leaf oil compounds on Pediculus capitis. J Agr Food Chem 51: 4884-4888. 